\section{AB0229 INFLUENCE OF RHEUMATOLOGIST IN PRIMARY CARE}

MC García Gómez, C Galisteo, MP Lisbona, M Sanmartí, M Castellanos, N Navarro, C Alegre. Rheumatology, Hospital Malalties Rheumàtiques, Barcelona, Spain

\subsection{6/annrheumdis-2001.777}

\section{Background}

Objectives To study if there are differences between the type and frequency of pathologies visited in a surgery of rheumatology hospital before and after the presence of rheumatologist in primary care (PC).

Methods Descriptive and retrospective study in the urban medium from Barcelona. We revised the historial clinic from first six months period in 1995 (without rheumatologist in PC) and 2000 (when the rheumatologist goes once per week to the primary care centre). We analysed the variables age, sex and principal diagnosis from all the first visits attended. We assembled the diagnosis in 10 groups: infectious arthritis (1), connective tissue disorders (2), crystal-related arthropathies (3), inflammatories arhropaties (4), osteoarthritis and related disorders (5), regional pain problems and soft tissue (6), muscular diseases (7), tumours and paraneoplastic syndromes in the musculoskeletal system (9) and others pathologies (10). For the statistic test we use the chisquared test to contrast of proportions and the Fisher's exact test when the expected effectives were lower than 5 .

Results In 1995 we have done 779 first visits (with 2.3\% of loses the fact is not to have the historial clinic) and from 2000, 411 (11\% of loses caused by the same matter). In 1995 the average age was 55 years (3-86), the distribution per sex was: 185 men (24\%) and 594 women (76\%), and per pathology: group 1, $2(0.2 \%)$, group $2,45(5.72 \%)$, group $3,18(2.3 \%)$, group 4,23 (2.9\%), group $5,453(58.1 \%)$, group $6,152(19.5 \%)$, group 7,1 $(0.12 \%)$, group $8,55(7 \%)$, group $9,4(0.5 \%)$, group 10,26 (3.3\%). In 2000 the average age was 55 years (5-87), the distribution per sex was:126 men (30\%) and 285 women (70\%), and per pathology: group $1,1(0.2 \%)$, group $2,38(9.2 \%)$, group 3 , $14(3.4 \%)$, group 4,21 (5.1\%), group 5, 200 (48.6\%), group 6 $89(21.6 \%)$, group 7,0 , group $8,31(7.5 \%)$, group $9,3(0.73 \%)$, group 10, 14(3.4\%). We found significant difference between both years in the groups $2(\mathrm{p}=0.025)$ and $5(\mathrm{p}=0.0002)$.

Conclusion The total numbers of first visits in the hospital done in 2000 were half less than in 1995 (above all in degeneration pahology), without being geographic and demographic variations. The incorporation of the rheumatologist in PC would improve the quality assistance in the hospital and also allow an early diagnosis from many rheumatic illness that would benefit from it.

\section{AB0230 A COMPUTER-AIDED DIAGNOSTIC SYSTEM WORKING FROM BIBLIOGRAPHICINFORMATION AND EXPERT ADVICE}

M Beurton-Aimar, JP Vernhes. 'ISPED-Epidemiology Research Center, University Bordeaux; ${ }^{2}$ Bordeaux Cedex, France

\subsection{6/annrheumdis-2001.778}

Background The improvement of medical informatics and the introduction of computers in all medical fields have modified the nature of the information manipulated by diagnosis support systems. Systems must be able to store information, to consult validated clinical tables, and to compare the results for several diseases. ${ }^{1}$ This suggests the production of strongly inter-connected process modules, sharing a common core of information.
We have to problem to solve. The first one is to build a system which can treat several types of information about diseases. Ordinary, such systems try to aggregate information and to compute the diagnosis with a new global procedure. We assume that it is better, in first, to analyse each type of information with each treatment associated to it and after that to aggregate the different results into a global advice. The second part of the problem is to conceive a data organisation which allows to store only one patient description, enough generic to work for all diagnosis procedures.

Objectives

Methods We have adopted an Object Oriented Design to create the Knowledge Data Base and to obtain an architecture which allows the collaboration between the treatment modules. ${ }^{2}$ The model of the Knowledge Data Base contains a generic object Description composed from three sets of signs: present signs, missing signs and information signs (to store non-pathological data as sex, age ldots). Both patient and disease descriptions inherit their structure from the object Description. This organisation ensures the consistency between the patient and disease descriptions and simplifies their comparison. Two methods of diagnosis are defined: evaluation of criteria lists issued from bibliography (as ARA criteria), and scoring calculus from an expert advice. The first one eliminates all diseases for which the patient have exclusion signs. If the patient presents all criteria of a disease, the diagnosis is validated, else the score calculus produced an ordered list of possible. So, we can have a final advice about the patient case which is an aggregation of the two processes.

Results The realised software contains a KBD with more than one hundred signs to define 13 diseases in inflammatory rheumatology. 132 records of patients who present one of these 13 rheumatic diseases, was analysed. 83 (62.9\%) patients presented a complete criteria list for one disease. The scoring method classified the right disease in the head of the list in $106(80.3 \%)$ cases, and the aggregation of the two methods leads to make the right diagnostic for $120(90.9 \%)$ cases.

We have shown that it is possible to conceive such hybrid system which can deal with several types of information and can compare diseases for which information is heterogeneous. For example criteria list is missing for three diseases. We think that this way produces better results than when we try to mix all information in only one type of value and after that to compute with.

\section{Conclusion}

\section{REFERENCES}

1 Beurton-Aimar M, Vernhes JP, Le Blanc B, Dehais J, Salamon R. Creating a knowledge base for diagnosis making in rheumatology. Revue du rhumatisme1997:11:762

2 Beurton-Aimar M, Le Blanc B, Vernhes JP. LADRI: a decision making system in inflammatory rheumatology. Proceedings of the 7th International Conference on Intelligent Systems, July 1-2, 1998, 51-6

\section{OP0106 EPIDEMIOLOGY OF HIP FRACTURE IN AGE GROUPS OF HIGH OSTEOPOROSIS RISK ON THE URAL, RUSSIA}

LI Kuzmina, OM Lesnyak. Rheumatologist, Ural State Medical Academy, Yekaterinburg, Russia

\subsection{6/annrheumdis-2001.779}

Background The epidemiology of hip fractures in Eastern parts of Russia has not yet been studied.

Objectives The aim of this retrospective study was to analyse the incidence of hip fracture in subjects over 50. 
Methods The total population of the city is 1,5 million population in the city of Yekaterinburg. The medical records of four city hospitals where patients with hip fractures were admitted as well as from outpatient emergency units were analysed for 1992-1997. The city administration provided statistical data on relevant population number and age distribution. Hip fracture has been defined as a fracture of the femoral neck or intertrochanteric region fracture (ICD IX code 820.0 and 820.2). There were no exclusion criteria other then the hip fracture occurring as a result of major trauma.

Results In the health care areas studied there were 2777 hip fractures: 599 males and 2178 females. The mean age of males was $69,0 \pm 11,2$ years of age and females $-76,5 \pm 9,3$ respectively $(\mathrm{p}<0,0001)$. The incidence of hip fracture for the examined period made up 132,3/100 000 population in those over 50 years $(76,9 / 100000$ for males and 164,8/100 000 for females). The ratio of male to females cases was $1: 2$. The incidence of hip fracture rose exponentially with age in both genders. The occurrence of hip fracture was maximal in subjects over 80 years old and made up 744,1/100 $000(476,9 / 100000$ males and 818,2/100 000 females). Male/female ratio was 1:1,7. The incidence of hip fracture in our population has not seasonal variation.

Conclusion More recent data showed significant differences in the incidence of hip fractures between Russian regions. The lowest rate of hip fracture was found in the western part of Russia and the highest in the Ural (Yekaterinburg). There was a large difference in incidence between regions, which suggests important environmental factors in the causation of hip fracture.

\section{OP0108 AGGRESSIVE LYMPHOMAS (DIFFUSE LARGE B CELL LYMPHOMAS) ARE OVERREPRESENTED IN RA PATIENTS}

${ }^{1} \mathrm{E}$ Baecklund, ${ }^{2} \mathrm{C}$ Sundström, ${ }^{3} \mathrm{~A}$ Ekbom, ${ }^{3} \mathrm{~N}$ Feltelius, ${ }^{3} \mathrm{~L}$ Klareskog. ${ }^{1}$ Department of Rheumatology; ${ }^{2}$ Department of Pathology, University Hospital, Uppsala; ${ }^{3}$ Department of Medicine, Karolinska Hospital, Stockholm, Sweden

\subsection{6/annrheumdis-2001.780}

Background Patients with rheumatoid arthritis (RA) have an increased risk to develop lymphomas. In a previous case-control study we found a strong association between high disease activity in RA and the risk of lymphoma.

To get a better understanding of the biological background of this connexion we examined lymphoma subtypes in RA patients. Objectives To study the distribution of lymphoma subtypes in RA patients compared to the general population.

Methods In a population-based cohort of 11,683 patients with RA in the Uppsala Health Care region in Sweden,42 cases of lymphoma were identified through record linkages with the Swedish cancer registry 1965 through 1984.

The medical records and paraffin embedded lymphoma tissues were collected.

The lymphomas were reclassified according to the recently described REAL classification. The distribution was compared to lymphoma subtypes in the general population according to the Non-Hodgkin's Lymphoma (NHL) Classification Project. ${ }^{1}$

Results One patient was excluded as the RA diagnosis was wrong. Tissues from 36 of the remaining patients were found and reviewed. NHL was found in 33 patients, Hodgkin's disease in 2 and 1 patient did not have lymphoma.

Compared with the expected there was a more than 2-fold increased number of diffuse large B-cell lymphoma (22 patients, $69 \%$ of NHL's compared with $31 \%$ expected).
Few of the patients had been treated with immunosuppressive drugs.

Conclusion The distribution of lymphoma subtypes in RA patients differs from the expected.

This supports the idea of a specific underlying mechanism behind lymphoma development in RA patients. This mechanism may also be of importance for RA development and activity. Genetics and other characteristics of those RA patients who develop lymphomas deserve further studies.

\section{REFERENCE}

1 The Non-Hodgkin's Lymphoma Classification Project: A Clinical Evaluation of the International Lymphoma Study Group. Blood 1997;89(11):3909-18

\section{OP0109 HIGHER INCIDENCE OF THROMBOEMBOLIC EVENTS AMONG PATIENTS WITH RHEUMATOID ARTHRITIS VS. OSTEOARTHRITIS, AND VS. NO ARTHRITIS, IN THE GENERAL PRACTICE RESEARCH DATABASE (GPRD)}

DJ Watson, T Rhodes. Epidemiology, Merck Research Laboratories, West Point, USA

\subsection{6/annrheumdis-2001.781}

Background Persons with Rheumatoid arthritis (RA) have been noted to have increased all-cause and cardiovascular (CV) disease mortality relative to those without RA. Data on mortality in patients with RA vs those with osteoarthritis (OA), and data on nonfatal + fatal CV incidence in patients with RA relative to those with $\mathrm{OA}$ and those with no arthritis are lacking.

Objectives To compare the incidence of all cause mortality, myocardial infarction (MI), sudden death (SD), cerebrovascular events (stroke; excluding transient ischaemic attack), CV death (fatal MI, sudden death, fatal stroke), and all thromboembolic events in RA patients compared with patients with OA but not RA, and compared with those with no arthritis, in the GPRD.

Methods The GPRD is a general practice research database that currently contains over 35 million patient years of data from the UK and represents 6\% of general practice patients in England and Wales. This was an observational cohort study of patients $>40$ years old from GPRD practices in which $>80 \%$ of patients had recorded visits. Patients with history of MI or stroke prior to beginning of follow-up were excluded. Person-time was calculated for each patient by gender, age (5 year intervals) and diagnosis (RA; OA but not RA, and no arthritis). The rate/1000 person-years was computed for each gender, age, and diagnosis stratum. For each patient, only the first incident endpoint was counted and attributed to the stratum in which it occurred. Gender and age-adjusted incidence rates and ratios of rates of each endpoint during person-time with RA vs that with OA but not RA, and vs that with no arthritis were computed using Poisson regression.

Results 594 practices contributed 6,533,474 patients to the analysis. The incidence of RA was $0.68 / 1000$ patient years in men and 1.33/1000 patient years in women. Age-adjusted rates of all endpoints were significantly increased for both men and women with RA compared to those with OA but not RA (except for sudden death, which was significantly increased in women only). Age- and gender-adjusted rate ratios (95\% CI) were: all-cause mortality 1.72 (1.67, 1.78); MI 1.32 (1.24, 1.41); SD 1.33 $(1.09,1.63)$; stroke 1.26 (1.18, 1.34); CV death 1.41 (1.24, 1.61); and all thromboembolic events 1.31 (1.25, 1.39). Similar results were seen when patients with RA were compared with those with no arthritis: age- and gender-adjusted rate ratios (95\% CI) with RA vs no arthritis were: all-cause mortality 1.60 